\title{
Delay-Dependent Stability Criteria of Uncertain Periodic Switched Recurrent Neural Networks with Time-Varying Delays
}

\author{
Xing Yin, ${ }^{1}$ Jun Li, ${ }^{2}$ Weigen $W_{u}{ }^{1}$ and Qiranrong $\operatorname{Tan}^{1}$ \\ ${ }^{1}$ College of Computer Science, Panzhihua University, Panzhihua 617000, China \\ 2 School of Computer Science and Technology, Nanjing University of Science and Technology, \\ Nanjing 219004, China \\ Correspondence should be addressed to Xing Yin, xingyin11@126.com
}

Received 20 June 2011; Revised 29 August 2011; Accepted 17 September 2011

Academic Editor: Baodong Zheng

Copyright (C) 2011 Xing Yin et al. This is an open access article distributed under the Creative Commons Attribution License, which permits unrestricted use, distribution, and reproduction in any medium, provided the original work is properly cited.

\begin{abstract}
This paper deals with the problem of delay-dependent stability criterion of uncertain periodic switched recurrent neural networks with time-varying delays. When uncertain discrete-time recurrent neural network is a periodic system, it is expressed as switched neural network for the finite switching state. Based on the switched quadratic Lyapunov functional approach (SQLF) and freeweighting matrix approach (FWM), some linear matrix inequality criteria are found to guarantee the delay-dependent asymptotical stability of these systems. Two examples illustrate the exactness of the proposed criteria.
\end{abstract}

\section{Introduction}

Recurrent neural networks (RNNs) are a very important tool for many application areas such as associative memory, pattern recognition, signal processing, model identification, and combinatorial optimization. With the development of research on RNNs in theory and application, the model is more and more complex. When the continuous-time RNNs are simulated using computer, they should be discretized into discrete-time RNNs [1-3]. Simultaneously, in implementations of artificial neural networks, time-varying delay may occur due to finite switching speeds of the amplifiers and communication time [4, 5]. Therefore, researchers have considered that discrete-time RNNs with time-varying delay are incorporated in the processing and/or transmission parts of the network architectures [6-9]. Parameter uncertainties and nonautonomous phenomena often exist in real systems due to modeling inaccuracies [4]. Particularly when we consider a long-term dynamical behaviors of the system and consider seasonality of the changing environment, the parameters of the system usually 
will change with time [10-14]. In order to model those systems with neural networks, the uncertain (or switched or jumping) neural networks with time-varying delay appear in many papers $[6,15-24]$. So in this paper we consider the stability of the following discrete-time recurrent neural networks with time-varying delay:

$$
\begin{aligned}
u(k+1)= & (A+\Delta A(k)) u(k)+\left(W_{1}+\Delta W_{1}(k)\right) g(u(k)) \\
& +\left(W_{2}+\Delta W_{2}(k)\right) f(u(k-d(k)))+(I+\Delta I(k)),
\end{aligned}
$$

where $u(k)=\left\{u_{1}(k), u_{2}(k), \ldots, u_{n}(k)\right\} \in R^{n}$ is the state vector associated with $n$ neurons, $A=$ $\operatorname{diag}\left\{a_{1}, a_{2}, \ldots, a_{n}\right\}$ is a diagonal matrix with positive entries, $W_{1}$ and $W_{2}$ are, respectively, the connection weight matrix and the delayed connection weight matrix, $I$ is input vector, $g(u)=\left\{g_{1}(u), g_{2}(u), \ldots, g_{n}(u)\right\}$ and $f(u)=\left\{f_{1}(u), f_{2}(u), \ldots, f_{n}(u)\right\}$ are the neuron activation function vectors, and $d(k)$ is nonnegative differential time-varying functions which denote the time delays and satisfy

$$
0 \leq d_{1} \leq d(k) \leq d_{2}
$$

In most literatures it is required that parameter uncertainty matrices, such as $\Delta A(k)$, $\Delta W_{1}(k), \Delta W_{2}(k)$, and $\Delta I(k)$, should be in the form

$$
\left[\Delta A(k) \Delta W_{1}(k) \Delta W_{2}(k) \Delta I(k)\right]=D F(k)\left[E_{a} E_{w 1} E_{w 2} E_{i}\right]
$$

where $E_{a}, E_{w 1}, E_{w 2}$, and $E_{i}$ are given constant matrices of appropriate dimensions and $F(k)$ is an uncertain matrix such that

$$
F^{T}(k) F(k) \leq I
$$

In practice, however, $\Delta A(k), \Delta W_{1}(k), \Delta W_{2}(k)$, and $\Delta I(k)$ are generally difficult to have the decomposition of matrices for $D, E_{a}, E_{w 1}, E_{w 2}$, and $E_{i}$. In addition, periodic oscillation in recurrent neural networks is an interesting dynamic behavior as many biological and cognitive activities require repetition [7, 10, 11, 25]. Simultaneously, periodic oscillations in recurrent neural networks have been found in many applications such as associative memories, pattern recognition, machine learning, and robot motion control [25]. So, if (1.1) is an uncertain periodic neural network in which the period is less than a constant $b$, then (1.1) can be expressed as switched neural network for the finite switching state, that is, if (1.1) is a neural network with period $a(0<a \leq b)$, then $\Delta A(k)=\Delta A(k+a), \Delta W_{1}(k)=\Delta W_{1}(k+a)$, $\Delta W_{2}(k)=\Delta W_{2}(k+a)$, and $\Delta I(k)=\Delta I(k+a)$, which is corresponding to a switched neural network set: $\Omega=\bigcup_{a=1}^{b} U_{a}$, where $U_{a}=\left\{S_{a i}=\left(A_{a i}, W_{1 a i}, W_{2 a i}, I_{a i}\right) \mid 0<i \leq a\right\}, A_{a i}=$ $A+\Delta A(k+i), W_{1 a i}=W_{1}+\Delta W_{1}(k+i), W_{2 a i}=W_{2}+\Delta W_{2}(k+i)$, and $I_{a i}=I+\Delta I(k+i)$. Suppose $N$ is the number of elements of $\Omega$; then (1.1) is actually modified by

$$
u(k+1)=A_{r(k)} u(k)+W_{1 r(k)} g(u(k))+W_{2 r(k)} f(u(k-d(k)))+I_{r(k)},
$$


where $r(k)$ is a switching rule defined by $r(k): N \rightarrow \Omega$ with $\Omega=\left\{S_{1}, S_{2}, \ldots, S_{N}\right\}$. Moreover, $r(k)=j$ means the sub-recurrent neural network (sub-RNN) $S_{j}$, which is corresponding to $\left(A_{j}, W_{1 j}, W_{2 j}, I_{j}\right)$, is active.

The dynamic behaviors of those models are foundations for applications. Under (1.5) most papers discuss the stability of uncertain neural networks with the common Lyapunov function approach $[5,6,15-23,25]$. To the best of the authors' knowledge, up to now, there is scarcely any paper that studies the uncertain periodic neural networks using the SQLF. This situation motivates this research.

Motivated by the above discussions, the authors intend to study a problem of the delay-dependent stability criterion of uncertain discrete-time recurrent neural networks with time-varying delays that the uncertain recurrent neural networks have a finite number of subRNNs, and the sub-RNNs may change from one to another according to arbitrary switching and restricted switching. The contributions of this paper are the following. (1) Using a switching graph, uncertain periodic recurrent neural networks with time-varying delays are transformed into switched recurrent neural networks; (2) the derivative of the SQLF (3.7) of the literature [8] is improved in (3.11), please see Remark 4.3 and Table 3; (3) based on the switching graph, the delay-dependent stability criteria of switched recurrent neural networks are studied by FWM and SQLF. Then an effective LMI approach is developed to solve the problem.

This paper is organized as follows. In Section 2, we give some basic definitions. We analyze the stability of the system (2.2) with the SQLF and FWM in Section 3. Some examples are given in Section 4. Section 5 offers the conclusions of this paper.

\section{Preliminaries}

In many electronic circuits, nonmonotonic functions can be more appropriate to describe the neuron activation in designing and implementing an artificial neural network [7]; hence, we have the following assumption.

For any $j \in\{1,2, \ldots, n\}$, there exist constants $g_{j}^{-}, g_{j}^{+}, f_{j}^{-}$, and $f_{j}^{+}$such that

$$
\begin{gathered}
g_{j}^{-} \leq \frac{g_{j}\left(\theta_{1}\right)-g_{j}\left(\theta_{2}\right)}{\theta_{1}-\theta_{2}} \leq g_{j}^{+}, \quad \forall \theta_{1}, \theta_{2} \in R, \\
f_{j}^{-} \leq \frac{f_{j}\left(\theta_{1}\right)-f_{j}\left(\theta_{2}\right)}{\theta_{1}-\theta_{2}} \leq f_{j}^{+}, \quad \forall \theta_{1}, \theta_{2} \in R .
\end{gathered}
$$

Under the assumption, the equilibrium points of UDNN (1.1) exist by the fixed point theorem [1]. In the following, let $u^{\circ}=\left\{u_{1}^{\circ}, u_{2}^{\circ}, \ldots, u_{n}^{\circ}\right\}$ be the equilibrium point of (1.1); then $x(\cdot)=u(\cdot)-u^{\circ}$. The systems (1.1) and (1.5) are, respectively, shifted to the following form:

$$
\begin{gathered}
x(k+1)=(A+\Delta A(k)) x(k)+\left(W_{1}+\Delta W_{1}(k)\right) g(x(k))+\left(W_{2}+\Delta W_{2}(k)\right) f(x(k-\tau(k))), \\
x(k+1)=A_{r(k)} x(k)+W_{1 r(k)} g(x(k))+W_{2 r(k)} f(x(k-d(k))), \quad r(k) \in \Omega .
\end{gathered}
$$

For convenience, the switching graph is defined. 
Definition 2.1. Let $\Gamma=(\Omega, \mathbf{W})$ be a switching graph, where $\Omega$ is the set of sub-RNNs $S_{i}$ and $\mathbf{W}$ is the set of weighted $\operatorname{arcs} \mathbf{w}_{i j} \in\{0,1\} . \mathbf{w}_{i j}=1$ (or 0 ) represents the sub-RNN $S_{i}$ switches (or does not switch) to the sub-RNN $S_{j}$.

Remark 2.2. When $\mathbf{w}_{i j}=1$, if $\sum_{l=1}^{N} \mathbf{w}_{j l}=0$, that is sub-RNN $S_{j}$ cannot switch to any other sub-RNN, we suppose that the uncertain neural networks will always stay in the sub-RNN $S_{i}$ that means $\mathbf{w}_{i i}=1$ and $\mathbf{w}_{i j}=0$.

Throughout this paper, the superscript $T$ stands for the transpose of a matrix, $P>0$ means that the matrix $P$ is positive definite, and the symmetric terms in a symmetric matrix are denoted by $*$, for example,

$$
\begin{gathered}
{\left[\begin{array}{ll}
M & O \\
* & N
\end{array}\right]=\left[\begin{array}{cc}
M & O \\
O^{T} & N
\end{array}\right],} \\
G_{1}=\operatorname{diag}\left\{g_{1}^{-} g_{1}^{+}, g_{2}^{-} g_{2}^{+}, \ldots, g_{n}^{-} g_{n}^{+}\right\}, \\
G_{2}=\operatorname{diag}\left\{-\frac{g_{1}^{-}+g_{1}^{+}}{2},-\frac{g_{2}^{-}+g_{2}^{+}}{2}, \ldots,-\frac{g_{n}^{-}+g_{n}^{+}}{2}\right\}, \\
F_{1}=\operatorname{diag}\left\{f_{1}^{-} f_{1}^{+}, f_{2}^{-} f_{2}^{+}, \ldots, f_{n}^{-} f_{n}^{+}\right\}, \\
F_{2}=\operatorname{diag}\left\{-\frac{f_{1}^{-}+f_{1}^{+}}{2},-\frac{f_{2}^{-}+f_{2}^{+}}{2}, \ldots,-\frac{f_{n}^{-}+f_{n}^{+}}{2}\right\} .
\end{gathered}
$$

\section{Asymptotical Stability of Uncertain Periodic Switched Recurrent Neural Networks}

Theorem 3.1. Let $d_{1}$ and $d_{2}$ be positive integers such that $0 \leq d_{1} \leq d_{2}$. Based on a switching graph $\Gamma$, the system (2.2) is asymptotical stable if, when $\mathbf{w}_{i j}=1\left(S_{i}, S_{j} \in \Omega\right)$, there exist the corresponding symmetric matrices $P_{i}=P_{i}^{T}>0, Q_{1}=Q_{1}^{T}>0, Q_{2}=Q_{2}^{T}>0, R=R^{T}>0, Z_{1}=Z_{1}^{T}>0$, $Z_{2}=Z_{2}^{T}>0, X^{i j}=\left(X^{i j}\right)^{T} \geq 0, U^{i j}=\left(U^{i j}\right)^{T} \geq 0, H^{i j}=\operatorname{diag}\left\{h_{1}^{i j}, h_{2}^{i j}, \ldots, h_{n}^{i j}\right\} \geq 0, O^{i j}=$ $\operatorname{diag}\left\{o_{1}^{i j}, o_{2}^{i j}, \ldots, o_{n}^{i j}\right\} \geq 0$, and any appropriate dimensional matrices $N^{i j}, M^{i j}$, and $T^{i j}$ such that the following LMIs hold:

$$
\begin{gathered}
\Phi^{i j}=\left[\begin{array}{cccccc}
\Phi_{11}^{i j} & \Phi_{12}^{i j} & \Phi_{13}^{i j} & \Phi_{14}^{i j} & \Phi_{15}^{i j} & \Phi_{16}^{i j} \\
* & \Phi_{22}^{i j} & \Phi_{23}^{i j} & \Phi_{24}^{i j} & 0 & 0 \\
* & * & \Phi_{33}^{i j} & \Phi_{34}^{i j} & 0 & 0 \\
* & * & * & \Phi_{44}^{i j} & 0 & -F_{2} O^{i j} \\
* & * & * & * & \Phi_{55}^{i j} & \Phi_{56}^{i j} \\
* & * & * & * & * & \Phi_{66}^{i j}
\end{array}\right]<0, \\
\Lambda_{1}^{i j}=\left[\begin{array}{cc}
X^{i j} & N^{i j} \\
* & Z_{1}
\end{array}\right]>0,
\end{gathered}
$$


Discrete Dynamics in Nature and Society

$$
\begin{gathered}
\Lambda_{2}^{i j}=\left[\begin{array}{cc}
U^{i j} & T^{i j} \\
* & Z_{2}
\end{array}\right]>0, \\
\Lambda_{3}^{i j}=\left[\begin{array}{cc}
X^{i j}+U^{i j} & M^{i j} \\
* & Z_{1}+Z_{2}
\end{array}\right]>0,
\end{gathered}
$$

where

$$
\begin{aligned}
\Phi_{11}^{i j}= & A_{i}^{T} P_{j} A_{i}-P_{i}+Q_{1}+Q_{2}+\left(d_{2}-d_{1}+1\right) R+\left(A_{i}-E\right)^{T}\left(d_{2} Z_{1 j}+\left(d_{2}-d_{1}\right) Z_{2 j}\right)\left(A_{i}-E\right) \\
& +\left(N_{1}^{i j}\right)^{T}+N_{1}^{i j}+d_{2} X_{11}^{i j}+\left(d_{2}-d_{1}\right) U_{11}^{i j}-G_{1} H^{i j}, \\
\Phi_{12}^{i j}= & \left(N_{2}^{i j}\right)^{T}+T_{1}^{i j}+d_{2} X_{12}^{i j}+\left(d_{2}-d_{1}\right) U_{12}^{i j} \\
\Phi_{13}^{i j}= & \left(N_{3}^{i j}\right)^{T}-M_{1}^{i j}+d_{2} X_{13}^{i j}+\left(d_{2}-d_{1}\right) U_{13^{\prime}}^{i j} \\
\Phi_{14}^{i j}= & -N_{1}^{i j}+\left(N_{4}^{i j}\right)^{T}+M_{1}^{i j}-T_{1}^{i j}+d_{2} X_{14}^{i j}+\left(d_{2}-d_{1}\right) U_{14^{\prime}}^{i j} \\
\Phi_{15}^{i j}= & A_{i}^{T} P_{j} W_{1 i}+\left(A_{i}-E\right)^{T}\left(d_{2} Z_{1}+\left(d_{2}-d_{1}\right) Z_{2}\right) W_{1 i}-G_{2} H^{i j}, \\
\Phi_{16}^{i j}= & A_{i}^{T} P_{j} W_{2 i}+\left(A_{i}-E\right)^{T}\left(d_{2} Z_{1}+\left(d_{2}-d_{1}\right) Z_{2}\right) W_{2 i}, \\
\Phi_{22}^{i j}= & -Q_{1}-R+\left(T_{2}^{i j}\right)^{T}+T_{2}^{i j}+d_{2} X_{22}^{i j}+\left(d_{2}-d_{1}\right) U_{22^{\prime}}^{i j} \\
\Phi_{23}^{i j}= & -M_{2}^{i j}+\left(T_{3}^{i j}\right)^{T}+d_{2} X_{23}^{i j}+\left(d_{2}-d_{1}\right) U_{23^{\prime}}^{i j} \\
\Phi_{24}^{i j}= & -N_{2}^{i j}+\left(T_{4}^{i j}\right)^{T}+M_{2}^{i j}-T_{2}^{i j}+d_{2} X_{24}^{i j}+\left(d_{2}-d_{1}\right) U_{24}^{i j}, \\
\Phi_{33}^{i j}= & -Q_{2}-R-M_{3}^{i j}-\left(M_{3}^{i j}\right)^{T}+d_{2} X_{33}^{i j}+\left(d_{2}-d_{1}\right) U_{33}^{i j}, \\
\Phi_{34}^{i j}= & -N_{3}^{i j}-\left(M_{4}^{i j}\right)^{T}+M_{3}^{i j}-T_{3}^{i j}+d_{2} X_{34}^{i j}+\left(d_{2}-d_{1}\right) U_{34}^{i j}, \\
\Phi_{44}^{i j}= & -N_{4}^{i j}-\left(N_{4}^{i j}\right)^{T}+M_{4}^{i j}+\left(M_{4}^{i j}\right)^{T}-T_{4}^{i j}-\left(T_{4}^{i j}\right)^{T}+d_{2} X_{44}^{i j}+\left(d_{2}-d_{1}\right) U_{44}^{i j}-F_{1} O^{i j}, \\
\Phi_{55}^{i j}= & W_{1 i}^{T} P_{j} W_{1 i}+W_{1 i}^{T}\left(d_{2} Z_{1}+\left(d_{2}-d_{1}\right) Z_{2}\right) W_{1 i}-H^{i j}, \\
\Phi_{56}^{i j}= & W_{1 i}^{T} P_{j} W_{2 i}+W_{1 i}^{T}\left(d_{2} Z_{1}+\left(d_{2}-d_{1}\right) Z_{2}\right) W_{2 i}, \\
\Phi_{66}^{i j}= & W_{2 i}^{T} P_{j} W_{2 i}+W_{2 i}^{T}\left(d_{2} Z_{1}+\left(d_{2}-d_{1}\right) Z_{2}\right) W_{2 i}-O^{i j} . \\
& \\
&
\end{aligned}
$$

Proof. Suppose that $y(l)=x(l+1)-x(l)$; then we have $x(k+1)=x(k)+y(k)$ and $x(k)=$ $x(k-d(k))+\sum_{i=k-d(k)}^{k-1} y(l)$. 
We consider the following SQLF:

$$
\begin{gathered}
V_{r(k)}(k)=V_{1 r(k)}(k)+V_{2 r(k)}(k)+V_{3 r(k)}(k)+V_{4 r(k)}(k), \\
V_{1 r(k)}(k)=x^{T}(k) P_{r(k)} x(k), \\
V_{2 r(k)}(k)=\sum_{l=k-d_{1}}^{k-1} x^{T}(l) Q_{1 r(k)} x(l)+\sum_{l=k-d_{2}}^{k-1} x^{T}(l) Q_{2 r(k)} x(l), \\
V_{3 r(k)}(k)=\sum_{\theta=k-d_{2}}^{k-d_{1}} \sum_{l=\theta}^{k-1} x^{T}(l) R_{r(k)} x(l), \\
V_{4 r(k)}(k)=\sum_{\theta=-d_{2}}^{-1} \sum_{l=k+\theta}^{k-1} y^{T}(l) Z_{1 r(k)} y(l)+\sum_{\theta=-d_{2}}^{-d_{1}-1} \sum_{l=k+\theta}^{k-1} y^{T}(l) Z_{2 r(k)} y(l) .
\end{gathered}
$$

It is clear that the following equations are true:

$$
\begin{aligned}
& \sum_{l=k-d_{2}}^{k-1} y^{T}(l) Z_{1 r(k)} y(l)=\sum_{l=k-d_{2}}^{k-d(k)-1} y^{T}(l) Z_{1 r(k)} y(l)+\sum_{l=k-d(k)}^{k-1} y^{T}(l) Z_{1 r(k)} y(l), \\
& \sum_{l=k-d_{2}}^{k-d_{1}-1} y^{T}(l) Z_{2 r(k)} y(l)=\sum_{l=k-d_{2}}^{k-d(k)-1} y^{T}(l) Z_{2 r(k)} y(l)+\sum_{l=k-d(k)}^{k-d_{1}-1} y^{T}(l) Z_{2 r(k)} y(l) .
\end{aligned}
$$

Firstly, we prove that under $\mathbf{w}_{i j}=1$ the SQLF is less than 0 . Suppose that $r(k)=i$ and $r(k+1)=j$, that means the sub-RNN $S_{i}$ switches to the sub-RNN $S_{j}$; we obtain

$$
\begin{aligned}
\Delta V_{1 i}(k)= & V_{1 j}(k+1)-V_{1 i}(k)=\left(A_{i} x(k)+W_{1 i} g(x(k))+W_{2 i} f(x(k-d(k)))\right)^{T} \\
& \times P_{j}\left(A_{i} x(k)+W_{1 i} g(x(k))+W_{2 i} f(x(k-d(k)))\right)-x^{T}(k) P_{i} x(k), \\
\Delta V_{2 i}(k)= & V_{2 j}(k+1)-V_{2 i}(k)=x^{T}(k)\left(Q_{1 j}+Q_{2 j}\right) x(k)-x^{T}\left(k-d_{1}\right) Q_{1 i} x\left(k-d_{1}\right) \\
& -x^{T}\left(k-d_{2}\right) Q_{2 i} x\left(k-d_{2}\right)+\sum_{l=k+1-d_{1}}^{k-1} x^{T}(l)\left(Q_{1 j}-Q_{1 i}\right) x(l) \\
& +\sum_{l=k+1-d_{2}}^{k-1} x^{T}(l)\left(Q_{2 j}-Q_{2 i}\right) x(l), \\
\Delta V_{3 i}(k)= & V_{3 j}(k+1)-V_{3 i}(k) \leq\left(d_{2}-d_{1}+1\right) x^{T}(k) R_{j} x(k) \\
& -x^{T}\left(k-d_{1}\right) R_{i} x\left(k-d_{1}\right)-x^{T}\left(k-d_{2}\right) R_{i} x\left(k-d_{2}\right)-x^{T}(k-d(k)) R_{i} x(k-d(k)) \\
& +\sum_{l=k+1-d_{1}}^{k-1} x^{T}(l)\left(R_{j}-R_{i}\right) x(l)+\sum_{\theta=k+1-d_{2}}^{k-d_{1}} \sum_{l=\theta}^{k-1} x^{T}(l)\left(R_{j}-R_{i}\right) x(l),
\end{aligned}
$$


Discrete Dynamics in Nature and Society

$$
\begin{aligned}
\Delta V_{4 i}(k)= & V_{4 j}(k+1)-V_{4 i}(k)=d_{2} y^{T}(k) Z_{1 j} y(k)+\left(d_{2}-d_{1}\right) y^{T}(k) Z_{2 j} y(k) \\
& -\sum_{l=k-d(k)}^{k-1} y^{T}(l) Z_{1 i} y(l)-\sum_{l=k-d(k)}^{k-d_{1}-1} y^{T}(l) Z_{2 i} y(l) \\
& -\sum_{l=k-d_{2}}^{k-d(k)-1} y^{T}(l)\left(Z_{1 i}+Z_{2 i}\right) y(l)+\sum_{\theta=-d_{2}}^{-1} \sum_{l=k+1+\theta}^{k-1} y^{T}(l)\left(Z_{1 j}-Z_{1 i}\right) y(l) \\
& +\sum_{\theta=-d_{2}}^{-d_{1}-1} \sum_{l=k+1+\theta}^{k-1} y^{T}(l)\left(Z_{2 j}-Z_{2 i}\right) y(l) .
\end{aligned}
$$

In order to strictly guarantee $\Delta V_{2 i}(k)<0, \sum_{l=k+1-d_{1}}^{k-1} x^{T}(l)\left(Q_{1 j}-Q_{1 i}\right) x(l)$ should be less than 0 . In the switching graph $\Gamma$ if there exists sub-RNN $S_{i}\left(S_{i} \in \Omega\right)$, which satisfied $\sum_{j=1}^{N} \mathbf{w}_{i j}=$ 0 , which means the $S_{i}$ cannot switch to any other sub-RNN, the equation $Q_{1 i}=Q_{1 j}$ can be grounded, otherwise the switching sequence must be $\beta=\left\{S_{\alpha_{1}} \rightarrow S_{\alpha_{2}} \rightarrow, \ldots, \rightarrow S_{\alpha_{j}} \rightarrow\right.$ $\left.\cdots, \rightarrow S_{\alpha_{L}} \rightarrow S_{\alpha_{L+1}}\right\}\left(S_{\alpha_{j}} \in \Omega\right)$, and there exist $l$ such that $S_{\alpha_{L+1}}=S_{\alpha_{l}}(1 \leq l<L)$. Because the affection of the sub-RNNs $S_{1}, S_{2} \ldots, S_{l-1}$ and $S_{l}$ on the whole system is before time $\alpha_{l}$, after $\alpha_{l}$ the $\beta$ changes to a periodic sequence $\beta^{\prime}=\left\{S_{\alpha_{l}} \rightarrow \cdots, \rightarrow S_{\alpha_{L}} \rightarrow S_{\alpha_{L+1}}\right\}$. Suppose that $i=\alpha_{l}=\alpha_{L+1}$; then in switching sequence $\beta^{\prime}$ the following LMIs all hold:

$$
\begin{gathered}
Q_{1 \alpha_{l}}-Q_{1 \alpha_{l+1}} \geq 0 \quad\left(Q_{1 i}-Q_{1(l+1)} \geq 0\right), \\
Q_{1 \alpha_{l+1}}-Q_{1 \alpha_{l+2}} \geq 0, \\
\vdots \\
Q_{1 \alpha_{L}}-Q_{1 \alpha_{L+1}} \geq 0 \quad\left(Q_{1(L)}-Q_{1 i} \geq 0\right) ;
\end{gathered}
$$

then the solution of (3.13) is $Q_{1 i}=Q_{1 \alpha_{l}}=Q_{1 \alpha_{l+1}}=\cdots=Q_{1 \alpha_{L}}$. Thus, we suppose that

$$
Q_{1}=Q_{1 i}=Q_{1 j}
$$

Similar to $\sum_{l=k+1-d_{1}}^{k-1} x^{T}(l)\left(Q_{1 j}-Q_{1 i}\right) x(l)$, together with $\sum_{l=k+1-d_{2}}^{k-1} x^{T}(l)\left(Q_{2 j}-Q_{2 i}\right) x(l), \sum_{l=k+1-d_{1}}^{k-1}$ $x^{T}(l)\left(R_{j}-R_{i}\right) x(l), \sum_{\theta=k+1-d_{2}}^{k-d_{1}} \sum_{l=\theta}^{k-1} x^{T}(l)\left(R_{j}-R_{i}\right) x(l), \sum_{\theta=-d_{2}}^{-1} \sum_{l=k+1+\theta}^{k-1} y^{T}(l)\left(Z_{1 j}-Z_{1 i}\right) y(l)$ and $\sum_{\theta=-d_{2}}^{-d_{1}-1} \sum_{l=k+1+\theta}^{k-1} y^{T}(l)\left(Z_{2 j}-Z_{2 i}\right) y(l)$, we suppose that

$$
\begin{gathered}
Q_{2}=Q_{2 i}=Q_{2 j}, \\
R=R_{i}=R_{j}, \\
Z_{1}=Z_{1 i}=Z_{1 j}, \\
Z_{2}=Z_{2 i}=Z_{2 j} .
\end{gathered}
$$


On the other hand, for any appropriately dimensioned matrices $N^{i j}, M^{i j}$, and $T^{i j}$ the following equations are true:

$$
\begin{gathered}
2 \xi^{T}(k) N^{i j}\left[x(k)-x(k-d(k))-\sum_{l=k-d(k)}^{k-1} y(l)\right]=0, \\
2 \xi^{T}(k) M^{i j}\left[x(k-d(k))-x\left(k-d_{2}\right)-\sum_{l=k-d_{2}}^{k-d(k)-1} y(l)\right]=0, \\
2 \xi^{T}(k) T^{i j}\left[x\left(k-d_{1}\right)-x(k-d(k))-\sum_{l=k-d(k)}^{k-d_{1}-1} y(l)\right]=0
\end{gathered}
$$

where $\xi(k)=\left[x^{T}(k), x^{T}\left(k-d_{1}\right), x^{T}\left(k-d_{2}\right), x^{T}(k-d(k))\right]^{T}$.

In addition, for any semipositive definite matrix $X^{i j}=\left(X^{i j}\right)^{T}$ and $U^{i j}=\left(U^{i j}\right)^{T}$, the following equations hold:

$$
\begin{gathered}
d_{2} \xi^{T}(k) X^{i j} \xi(k)-\sum_{l=k-d_{2}}^{k-d(k)-1} \xi^{T}(k) X^{i j} \xi(k)-\sum_{l=k-d(k)}^{k-1} \xi^{T}(k) X^{i j} \xi(k)=0, \\
\left(d_{2}-d_{1}\right) \xi^{T}(k) U^{i j} \xi(k)-\sum_{l=k-d_{2}}^{k-d(k)-1} \xi^{T}(k) U^{i j} \xi(k)-\sum_{l=k-d(k)}^{k-d_{1}-1} \xi^{T}(k) U^{i j} \xi(k)=0 .
\end{gathered}
$$

From the assumption, we have

$$
\begin{gathered}
\left(g_{l}(x(k))-g_{l}^{-} x_{l}(k)\right) \times\left(g_{l}(x(k))-g_{l}^{+} x_{l}(k)\right) \leq 0, \quad l=1,2, \ldots, n, \\
\left(f_{l}\left(x\left(k-d_{k}\right)\right)-f_{l}^{-} x_{l}\left(k-d_{k}\right)\right) \times\left(f_{l}\left(x\left(k-d_{k}\right)\right)-f_{l}^{+} x_{l}\left(k-d_{k}\right)\right) \leq 0, \quad l=1,2, \ldots, n .
\end{gathered}
$$

Similar to the conclusion in [8], for $H^{i j}=\operatorname{diag}\left\{h_{1}, h_{2}, \ldots, h_{n}\right\} \geq 0, O^{i j}=\operatorname{diag}\left\{o_{1}\right.$, $\left.o_{2}, \ldots, o_{n}\right\} \geq 0$ the following inequalities are also true:

$$
\begin{gathered}
-\left[\begin{array}{c}
x(k) \\
g(x(k))
\end{array}\right]^{T}\left[\begin{array}{cc}
G_{1} H^{i j} & G_{2} H^{i j} \\
\star & H^{i j}
\end{array}\right]\left[\begin{array}{c}
x(k) \\
g(x(k))
\end{array}\right] \geq 0, \\
-\left[\begin{array}{c}
x(k-d(k)) \\
f(x(k-d(k)))
\end{array}\right]^{T}\left[\begin{array}{cc}
F_{1} O^{i j} & F_{2} O^{i j} \\
\star & O^{i j}
\end{array}\right]\left[\begin{array}{c}
x(k-d(k)) \\
f(x(k-d(k)))
\end{array}\right] \geq 0 .
\end{gathered}
$$


Then we add the terms on the right side of (3.16)-(3.23) to yield

$$
\begin{aligned}
\Delta V_{i}(k)= & \Delta V_{1 i}(k)+\Delta V_{2 i}(k)+\Delta V_{3 i}(k)+\Delta V_{4 i}(k) \\
\leq & \eta_{1}^{T}(k) \Phi^{i j} \eta_{1}(k)-\sum_{l=k-d(k)}^{k-1} \eta_{2}^{T}(k, l) \Lambda_{1}^{i j} \eta_{2}(k, l) \\
& -\sum_{l=k-d(k)}^{k-d_{1}-1} \eta_{2}^{T}(k, l) \Lambda_{2}^{i j} \eta_{2}(k, l)-\sum_{l=k-d_{2}}^{k-d(k)-1} \eta_{2}^{T}(k, l) \Lambda_{3}^{i j} \eta_{2}(k, l),
\end{aligned}
$$

where $\eta_{1}^{T}(k)=\left[\xi^{T}(k), g^{T}(x(k)), f^{T}(x(k-d(k)))\right]^{T}, \eta_{2}^{T}(k, l)=\left[\xi^{T}(k), y^{T}(l)\right]^{T}$.

And $\xi(k)$ is defined in (3.18). $\Phi^{i j}, \Lambda_{1}^{i j}, \Lambda_{2}^{i j}$, and $\Lambda_{3}^{i j}$ are defined in (3.1)-(3.4). Therefore, when the corresponding LMIs satisfy $\Phi^{i j}<0, \Lambda_{1}^{i j} \geq 0, \Lambda_{2}^{i j} \geq 0$, and $\Lambda_{3}^{i j} \geq 0, S_{i}, S_{j} \in \Omega$, $\Delta V_{i}(k) \leq 0$.

Secondly, based on the switching graph $\Gamma$, when $\mathbf{w}_{i j}=1\left(S_{i}, S_{j} \in \Omega\right)$, all corresponding $\Delta V_{i}(k)$ are less than 0 that means the system (2.2) is asymptotical stable. This completes the proof of Theorem 3.1.

Remark 3.2. Using the method in [8], it is easily to know that the system is the globally exponentially stable.

Remark 3.3. In $V_{3 i}(k)$ of the literature [8], $-\sum_{l=k-d_{2}}^{k-d(k)} x^{T}(l) R_{i} x(l) \leq-x^{T}(k-d(k)) R_{i} x(k-d(k))$ may lead to considerable conservativeness. Then, it is improved as $-\sum_{l=k-d_{2}}^{k-d(k)} x^{T}(l) R_{i} x(l) \leq$ $-x^{T}\left(k-d_{2}\right) R_{i} x\left(k-d_{2}\right)-x^{T}(k-d(k)) R_{i} x(k-d(k))-x^{T}\left(k-d_{1}\right) R_{i} x\left(k-d_{1}\right)$. Please see Table 3.

Combined with Theorem 3.1, we consider the common Lyapunov function approach; then we have the following.

Corollary 3.4. Let $d_{1}$ and $d_{2}$ be positive integers such that $0 \leq d_{1} \leq d_{2}$. The system (2.2) is asymptotical stable if there exist symmetric matrices $P=P^{T}>0, Q_{1}=Q_{1}^{T}>0, Q_{2}=Q_{2}^{T}>0, R=$ $R^{T}>0, Z_{1}=Z_{1}^{T}>0, Z_{2}=Z_{2}^{T}>0, X=X^{T} \geq 0, U=U^{T} \geq 0, H=\operatorname{diag}\left\{h_{1}, h_{2}, \ldots, h_{n}\right\} \geq 0$, $O=\operatorname{diag}\left\{o_{1}, o_{2}, \ldots, o_{n}\right\} \geq 0$, and any appropriate dimensional matrices $N, M$, and $T$ such that the following LMIs hold:

$$
\begin{gathered}
\Phi_{i}=\left[\begin{array}{cccccc}
\Phi_{11} & \Phi_{12} & \Phi_{13} & \Phi_{14} & \Phi_{15} & \Phi_{16} \\
* & \Phi_{22} & \Phi_{23} & \Phi_{24} & 0 & 0 \\
* & * & \Phi_{33} & \Phi_{34} & 0 & 0 \\
* & * & * & \Phi_{44} & 0 & -F_{2} O \\
* & * & * & * & \Phi_{55} & \Phi_{56} \\
* & * & * & * & * & \Phi_{66}
\end{array}\right]<0, \quad S_{i} \in \Omega, \\
\Lambda_{1}=\left[\begin{array}{cc}
X & N \\
* & Z_{1}
\end{array}\right]>0 \\
\Lambda_{2}=\left[\begin{array}{cc}
U & T \\
* & Z_{2}
\end{array}\right]>0 \\
\Lambda_{3}=\left[\begin{array}{cc}
X+U & M \\
* & Z_{1}+Z_{2}
\end{array}\right]>0,
\end{gathered}
$$


where

$$
\begin{aligned}
\Phi_{11}= & A_{i}^{T} P A_{i}-P+Q_{1}+Q_{2}+\left(d_{2}-d_{1}+1\right) R+\left(A_{i}-E\right)^{T}\left(d_{2} Z_{1}+\left(d_{2}-d_{1}\right) Z_{2}\right)\left(A_{i}-E\right) \\
& +N_{1}^{T}+N_{1}+d_{2} X_{11}+\left(d_{2}-d_{1}\right) U_{11}-G_{1} H, \\
\Phi_{12}= & N_{2}^{T}+T_{1}+d_{2} X_{12}+\left(d_{2}-d_{1}\right) U_{12}, \\
\Phi_{13}= & N_{3}^{T}-M_{1}+d_{2} X_{13}+\left(d_{2}-d_{1}\right) U_{13} \\
\Phi_{14}= & -N_{1}+N_{4}^{T}+M_{1}-T_{1}+d_{2} X_{14}+\left(d_{2}-d_{1}\right) U_{14}, \\
\Phi_{15}= & A_{i}^{T} P W_{1 i}+\left(A_{i}-E\right)^{T}\left(d_{2} Z_{1}+\left(d_{2}-d_{1}\right) Z_{2}\right) W_{1 i}-G_{2} H, \\
\Phi_{16}= & A_{i}^{T} P W_{2 i}+\left(A_{i}-E\right)^{T}\left(d_{2} Z_{1}+\left(d_{2}-d_{1}\right) Z_{2}\right) W_{2 i}, \\
\Phi_{22}= & -Q_{1}-R+T_{2}^{T}+T_{2}+d_{2} X_{22}+\left(d_{2}-d_{1}\right) U_{22}, \\
\Phi_{23}= & -M_{2}+T_{3}^{T}+d_{2} X_{23}+\left(d_{2}-d_{1}\right) U_{23} \\
\Phi_{24}= & -N_{2}+T_{4}^{T}+M_{2}-T_{2}+d_{2} X_{24}+\left(d_{2}-d_{1}\right) U_{24}, \\
\Phi_{33}= & -Q_{2}-R-M_{3}-M_{3}^{T}+d_{2} X_{33}+\left(d_{2}-d_{1}\right) U_{33}, \\
\Phi_{34}= & -N_{3}-M_{4}^{T}+M_{3}-T_{3}+d_{2} X_{34}+\left(d_{2}-d_{1}\right) U_{34}, \\
\Phi_{44}= & -N_{4}-N_{4}^{T}+M_{4}+M_{4}^{T}-T_{4}-T_{4}^{T}-F_{1} O+d_{2} X_{44}+\left(d_{2}-d_{1}\right) U_{44}-R, \\
\Phi_{55}= & W_{1 i}^{T} P W_{1 i}+W_{1 i}^{T}\left(d_{2} Z_{1}+\left(d_{2}-d_{1}\right) Z_{2}\right) W_{1 i}-H, \\
\Phi_{56}= & W_{1 i}^{T} P W_{2 i}+W_{1 i}^{T}\left(d_{2} Z_{1}+\left(d_{2}-d_{1}\right) Z_{2}\right) W_{2 i}, \\
\Phi_{66}= & W_{2 i}^{T} P W_{2 i}+W_{2 i}^{T}\left(d_{2} Z_{1}+\left(d_{2}-d_{1}\right) Z_{2}\right) W_{2 i}-O .
\end{aligned}
$$

\section{Examples}

Example 4.1. Consider the discrete-time recurrent neural network (2.2) with

$$
\begin{aligned}
& A_{1}=\left[\begin{array}{ccc}
0.7 & 0 & 0 \\
0 & 0.2 & 0 \\
0 & 0 & 0.5
\end{array}\right], \quad W_{11}=\left[\begin{array}{ccc}
0.1 & -0.2 & 0.1 \\
0.3 & -0.1 & 0 \\
0 & -0.1 & -0.4
\end{array}\right], \quad W_{21}=\left[\begin{array}{ccc}
-0.3 & 0.1 & -0.2 \\
0.2 & -0.1 & 0.1 \\
0 & -0.02 & 0.07
\end{array}\right], \\
& A_{2}=\left[\begin{array}{ccc}
0.1 & 0 & 0 \\
0 & 0.2 & 0 \\
0 & 0 & 0.4
\end{array}\right], \quad W_{12}=\left[\begin{array}{ccc}
0.4 & -0.2 & 0 \\
0.2 & -0.2 & 0 \\
-0.3 & -0.1 & -0.5
\end{array}\right], \quad W_{22}=\left[\begin{array}{ccc}
-0.3 & 0.1 & -0.2 \\
0 & -0.03 & 0 \\
0.5 & -0.2 & 0.5
\end{array}\right] \text {, } \\
& A_{3}=\left[\begin{array}{ccc}
0.1 & 0 & 0 \\
0 & 0.4 & 0 \\
0 & 0 & 0.4
\end{array}\right], \quad W_{13}=\left[\begin{array}{ccc}
0.2 & 0 & 0.3 \\
0.04 & -0.3 & 0 \\
-0.1 & -0.6 & -0.1
\end{array}\right], \quad W_{23}=\left[\begin{array}{ccc}
-0.08 & 0.1 & -0.2 \\
0.2 & -0.4 & -0.05 \\
0 & -0.2 & -0.1
\end{array}\right] \text {, } \\
& \Gamma=\left[\begin{array}{lll}
0 & 1 & 0 \\
0 & 0 & 1 \\
1 & 0 & 0
\end{array}\right]
\end{aligned}
$$




$$
\begin{aligned}
& g_{1}(x)=\tanh (0.4 x), \quad g_{2}(x)=\tanh (0.2 x), \quad g_{3}(x)=\tanh (-0.8 x), \\
& f_{1}(x)=\tanh (-0.6 x), \quad f_{2}(x)=\tanh (0.4 x), \quad f_{3}(x)=\tanh (0.2 x) \text {. }
\end{aligned}
$$

Then we have

$$
G_{1}=F_{1}=\left[\begin{array}{lll}
0 & 0 & 0 \\
0 & 0 & 0 \\
0 & 0 & 0
\end{array}\right], \quad G_{2}=\left[\begin{array}{ccc}
0.2 & 0 & 0 \\
0 & 0.1 & 0 \\
0 & 0 & -0.4
\end{array}\right], \quad F_{2}=\left[\begin{array}{ccc}
0.3 & 0 & 0 \\
0 & -0.2 & 0 \\
0 & 0 & -0.1
\end{array}\right] \text {. }
$$

Employing the LMIs in Theorem 3.1 yields upper bounds on $d_{2}$ that guarantee the stability of system (1.1) for various lower bounds $d_{1}$, which are listed in Table 1 . When $d_{1}=1$ and $d_{2}=3$, it can be seen from Figure 1 that all the state solutions corresponding to the 10 random initial points are convergent asymptotically to the unique equilibrium $x^{*}=\{0,0,0\}$, and according to Theorem 3.1, LMIs (3.1)-(3.4) are solvable in Matlab 7.0.1.

Example 4.2. Consider the discrete-time recurrent neural network (2.2) with

$$
\begin{gathered}
A=\left[\begin{array}{ccc}
0.1 & 0 & 0 \\
0 & 0.2 & 0 \\
0 & 0 & 0.3
\end{array}\right], \quad W_{1}=\left[\begin{array}{ccc}
0.5 & -0.2 & 0.2 \\
0.1 & -0.1 & 0 \\
-0.4 & -0.1 & -0.3
\end{array}\right], \quad W_{2}=\left[\begin{array}{ccc}
-0.2 & 0.1 & 0.2 \\
0 & -0.3 & 0.1 \\
0 & -0.3 & 0.03
\end{array}\right], \\
g_{1}(x)=f_{1}(x)=\tanh (0.6 x), \quad g_{2}(x)=f_{2}(x)=\tanh (0.4 x), \quad g_{3}(x)=f_{3}(x)=\tanh (0.2 x) .
\end{gathered}
$$

Then we have

$$
G_{1}=F_{1}=\left[\begin{array}{lll}
0 & 0 & 0 \\
0 & 0 & 0 \\
0 & 0 & 0
\end{array}\right], \quad G_{2}=F_{2}=\left[\begin{array}{ccc}
0.3 & 0 & 0 \\
0 & 0.2 & 0 \\
0 & 0 & 0.1
\end{array}\right]
$$

Employing the LMIs in [8] and those in Corollary 3.4 yields upper bounds on $d_{2}$ that guarantee the stability of system for various lower bounds $d_{1}$, which are listed in Table 2 . It is clear that the obtained upper bounds of this paper are better than those of [8]. It can be seen from Figure 2 that, when $d_{1}=1$ and $d_{2}=3$, all the state solutions corresponding to the 10 random initial points are convergent asymptotically to the unique equilibrium $x^{*}=\{0,0,0\}$.

Remark 4.3. Employing the LMIs in [8] and those in Corollary 3.4 yields upper bounds on $d_{2}$ that guarantee the stability of system (1.1) of the Example 1 of [8] for various lower bounds $d_{1}$, which are listed in Table 3 . 


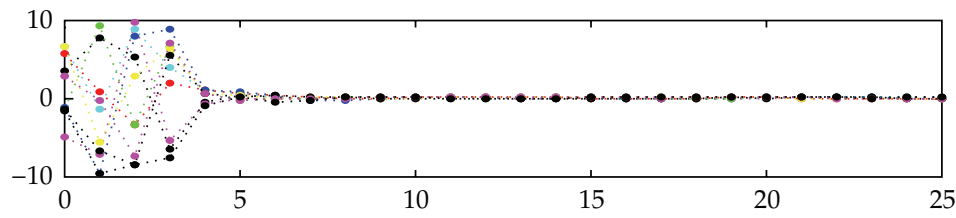

.... $x_{1}$

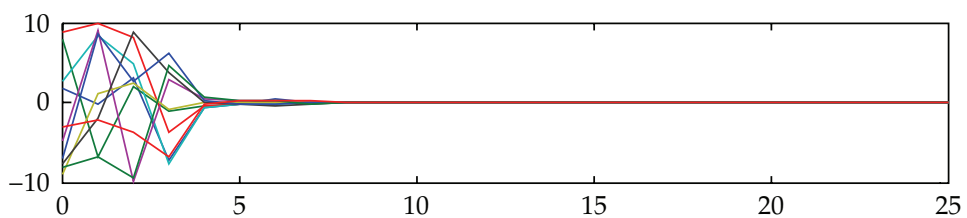

$x_{2}$

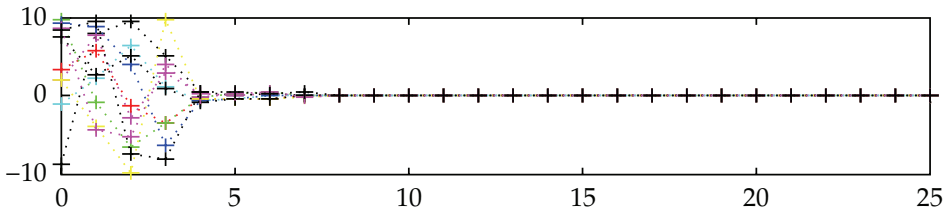

$+\cdot \cdot x_{3}$

Figure 1: Global convergence of states $x_{1}, x_{2}$, and $x_{3}$ in Example 4.1, when $d_{1}=1$ and $d_{2}=3$.

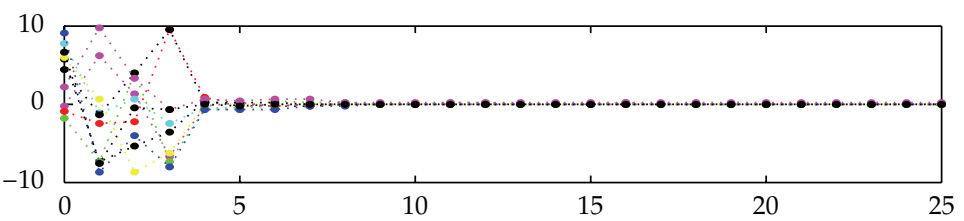

..... $x_{1}$

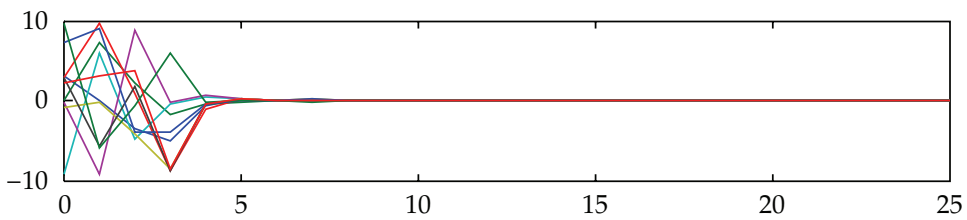

$-x_{2}$

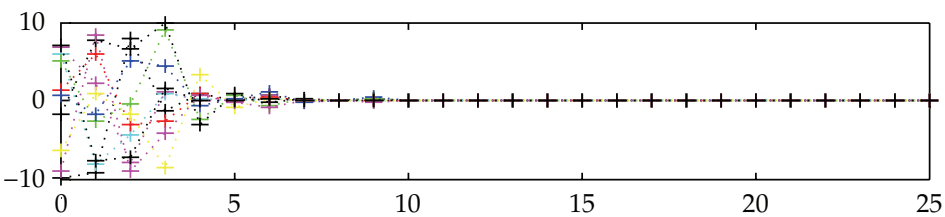

$\cdot+\cdot x_{3}$

Figure 2: Global convergence of states $x_{1}, x_{2}$, and $x_{3}$ in Example 4.2, when $d_{1}=1$ and $d_{2}=3$. 
Table 1: Allowable upper bound of $d_{2}$ with given $d_{1}$.

\begin{tabular}{lcccccc}
\hline$d_{1}$ & 0 & 2 & 4 & 6 & 8 & 20 \\
Theorem 3.1 & 9 & 11 & 13 & 15 & 17 & 29 \\
\hline
\end{tabular}

Table 2: Allowable upper bound of $d_{2}$ with given $d_{1}$.

\begin{tabular}{lccccccc}
\hline$d_{1}$ & 0 & 2 & 4 & 6 & 8 & 10 & 20 \\
Reference [8] & 20 & 22 & 24 & 26 & 28 & 30 & 40 \\
Corollary 3.4 & 38 & 40 & 42 & 44 & 46 & 48 & 58 \\
\hline
\end{tabular}

Table 3: Allowable upper bound of $d_{2}$ with given $d_{1}$.

\begin{tabular}{lccccc}
\hline$d_{1}$ & 0 & 2 & 4 & 6 & 10 \\
Reference [8] & 11 & 13 & 15 & 17 & 21 \\
Corollary 3.4 & 12 & 14 & 16 & 18 & 22 \\
\hline
\end{tabular}

\section{Conclusions}

This paper was dedicated to the delay-dependent stability of uncertain periodic switched recurrent neural networks with time-varying delay. A less conservative LMI-based globally stability criterion is obtained with the switched quadratic Lyapunov functional approach and free-weighting matrix approach for periodic uncertain discrete-time recurrent neural networks with a time-varying delay. One example illustrates the exactness of the proposed criterion. Another example demonstrates that the proposed method is an improvement over the existing one.

\section{Acknowledgments}

This work was supported by the Sichuan Science and Technology Department under Grant 2011JY0114. The authors would like to thank the Associate Editor and the anonymous reviewers for their detailed comments and valuable suggestions which greatly contributed to this paper.

\section{References}

[1] Y. Liu, Z. Wang, A. Serrano, and X. Liu, "Discrete-time recurrent neural networks with time-varying delays: exponential stability analysis," Physics Letters Section A, vol. 362, no. 5-6, pp. 480-488, 2007.

[2] Q.Song and Z. Wang, "A delay-dependent LMI approach to dynamics analysis of discrete-time recurrent neural networks with time-varying delays," Physics Letters Section A, vol. 368, no. 1-2, pp. 134$145,2007$.

[3] B. Zhang, S. Xu, and Y. Zou, "Improved delay-dependent exponential stability criteria for discretetime recurrent neural networks with time-varying delays," Neurocomputing, vol. 72, no. 1-3, pp. 321$330,2008$.

[4] H. Huang, G. Feng, and J. Cao, "Robust state estimation for uncertain neural networks with timevarying delay," IEEE Transactions on Neural Networks, vol. 19, no. 8, pp. 1329-1339, 2008.

[5] Y. Shen and J. Wang, "An improved algebraic criterion for global exponential stability of recurrent neural networks with time-varying delays," IEEE Transactions on Neural Networks, vol. 19, no. 3, pp. 528-531, 2008.

[6] Q. Song and J. Cao, "Global dissipativity on uncertain discrete-time neural networks with time-varying delays," Discrete Dynamics in Nature and Society, vol. 2010, Article ID 810408, 19 pages, 2010. 
[7] Y. Liu, Z. Wang, and X. Liu, "Asymptotic stability for neural networks with mixed time-delays: the discrete-time case," Neural Networks, vol. 22, no. 1, pp. 67-74, 2009.

[8] M. Wu, F. Liu, P. Shi, Y. He, and R. Yokoyama, "Improved free-weighting matrix approach for stability analysis of discrete-time recurrent neural networks with time-varying delay," IEEE Transactions on Circuits and Systems II, vol. 55, no. 7, pp. 690-694, 2008.

[9] C. Li, S. Wu, G. G. Feng, and X. Liao, "Stabilizing effects of impulses in discrete-time delayed neural networks," IEEE Transactions on Neural Networks, vol. 22, no. 2, pp. 323-329, 2011.

[10] H. Xiang, K. M. Yan, and B. Y. Wang, "Existence and global exponential stability of periodic solution for delayed high-order Hopfield-type neural networks," Physics Letters Section A, vol. 352, no. 4-5, pp. 341-349, 2006.

[11] H. Xiang, K. M. Yan, and B. Y. Wang, “Existence and global stability of periodic solution for delayed discrete high-order Hopfield-type neural networks," Discrete Dynamics in Nature and Society, vol. 2005, no. 3, pp. 281-297, 2005.

[12] G. Shaikhet and L. Shaikhet, "Stability of stochastic linear difference equations with varying delay," in Advances in Systems, Signals, Control and Computers, V. Bajic, Ed., pp. 101-104, IAAMSAD and SA branch of the Academy of Nonlinear Sciences, Durban, South Africa, 1998.

[13] J. Li, Y. Diao, M. Li, and X. Yin, "Stability analysis of Discrete Hopfield neural networks with the nonnegative definite monotone increasing weight function matrix," Discrete Dynamics in Nature and Society, vol. 2009, Article ID 673548, 2009.

[14] J. Li, Y. Diao, J. Mao, Y. Zhang, and X. Yin, "Stability analysis of discrete hopfield neural networks with weight function matrix," Lecture Notes in Computer Science, vol. 5370, pp. 760-768, 2008.

[15] X. Wu, Y. Wang, L. Huang, and Y. Zuo, "Robust exponential stability criterion for uncertain neural networks with discontinuous activation functions and time-varying delays," Neurocomputing, vol. 73, no. 7-9, pp. 1265-1271, 2010.

[16] H. Wu, W. Feng, and X. Liang, “New stability criteria for uncertain neural networks with interval time-varying delays," Cognitive Neurodynamics, vol. 2, no. 4, pp. 363-370, 2008.

[17] C. Y. Lu, H. H. Tsai, T. J. Su, J. S. H. Tsai, and C. W. Liao, “A delay-dependent approach to passivity analysis for uncertain neural networks with time-varying Delayd," Neural Processing Letters, vol. 27, no. 3, pp. 237-246, 2008.

[18] X. Lou and B. Cui, "Delay-dependent criteria for global robust periodicity of uncertain switched recurrent neural networks with time-varying delay," IEEE Transactions on Neural Networks, vol. 19, no. 4, pp. 549-557, 2008.

[19] J. Liang, Z. Wang, Y. Liu, and X. Liu, "Robust synchronization of an array of coupled stochastic discrete-time delayed neural networks," IEEE Transactions on Neural Networks, vol. 19, no. 11, pp. 1910 1921, 2008.

[20] H. Zhang and Y. Wang, "Stability analysis of Markovian jumping stochastic Cohen-Grossberg neural networks with mixed time delays," IEEE Transactions on Neural Networks, vol. 19, no. 2, pp. 366-370, 2008.

[21] Z. Wang, Y. Liu, and X. Liu, "State estimation for jumping recurrent neural networks with discrete and distributed delays," Neural Networks, vol. 22, no. 1, pp. 41-48, 2009.

[22] J. Zhou, Q. Song, and J. Yang, "Stochastic passivity of uncertain neural networks with time-varying delays," Abstract and Applied Analysis, vol. 2009, Article ID 725846, 16 pages, 2009.

[23] E. Zhu, Y. Wang, Y. Wang, H. Zhang, and J. Zou, "Stability analysis of recurrent neural networks with random delay and markovian switching," Journal of Inequalities and Applications, vol. 2010, Article ID 191546, 2010.

[24] J. Li, W. Wu, J. Yuan, Q. Tan, and X. Yin, “Delay-dependent stability criterion of arbitrary switched linear systems with time-varying delay," Discrete Dynamics in Nature and Society, vol. 2010, Article ID 347129, 16 pages, 2010.

[25] B. Chen and J. Wang, "Global exponential periodicity of a class of recurrent neural networks with oscillating parameters and time-varying delays," IEEE Transactions on Neural Networks, vol. 16, no. 6, pp. 1440-1448, 2005. 


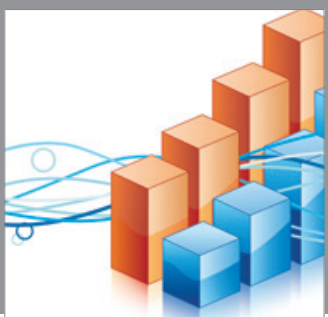

Advances in

Operations Research

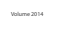

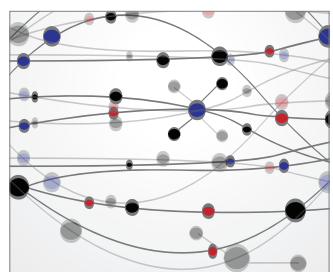

\section{The Scientific} World Journal
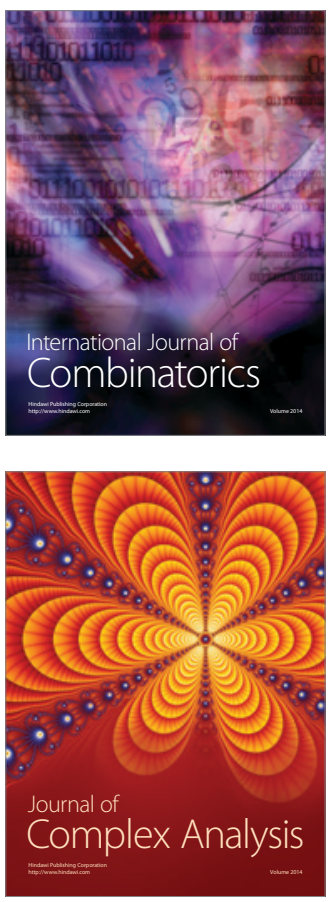

International Journal of

Mathematics and

Mathematical

Sciences
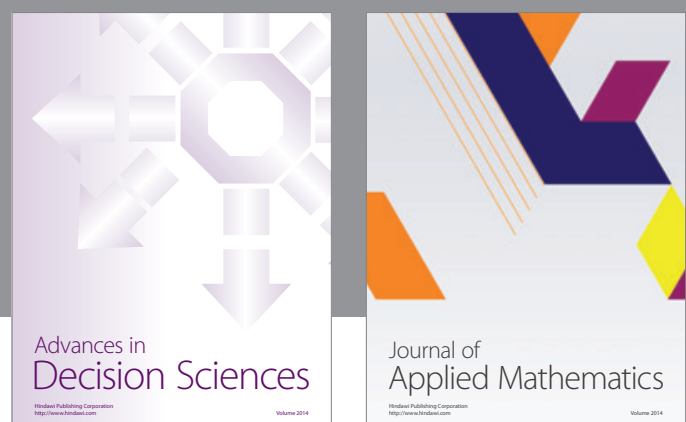

Journal of

Applied Mathematics
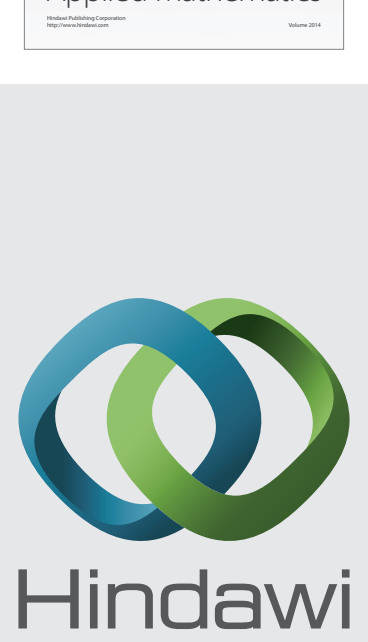

Submit your manuscripts at http://www.hindawi.com
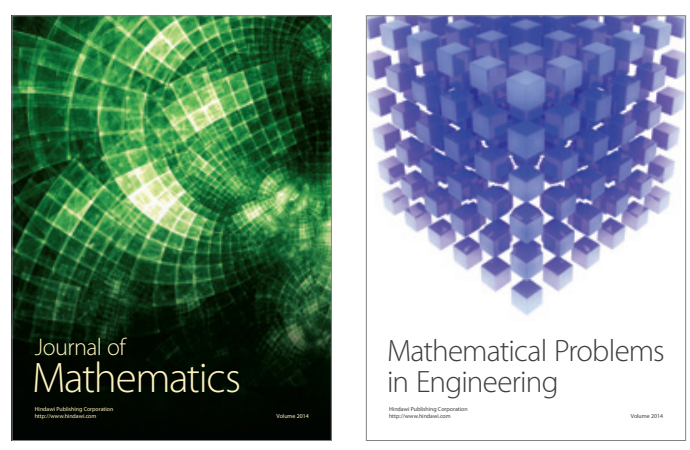

Mathematical Problems in Engineering
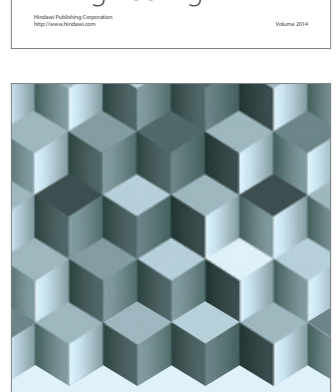

Journal of

Function Spaces
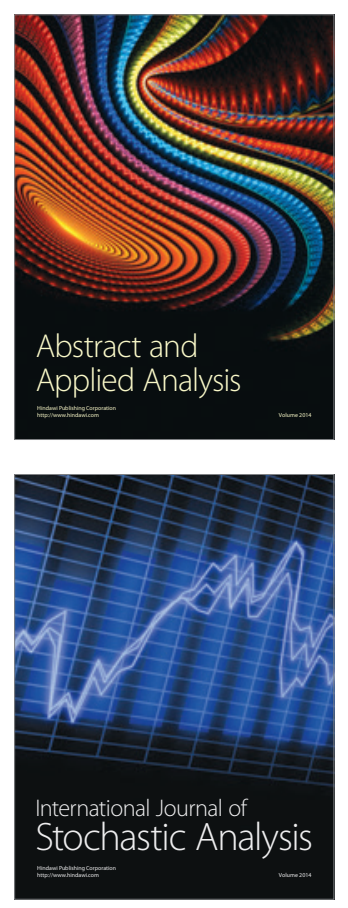

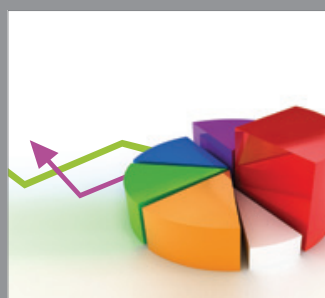

ournal of

Probability and Statistics

Promensencen
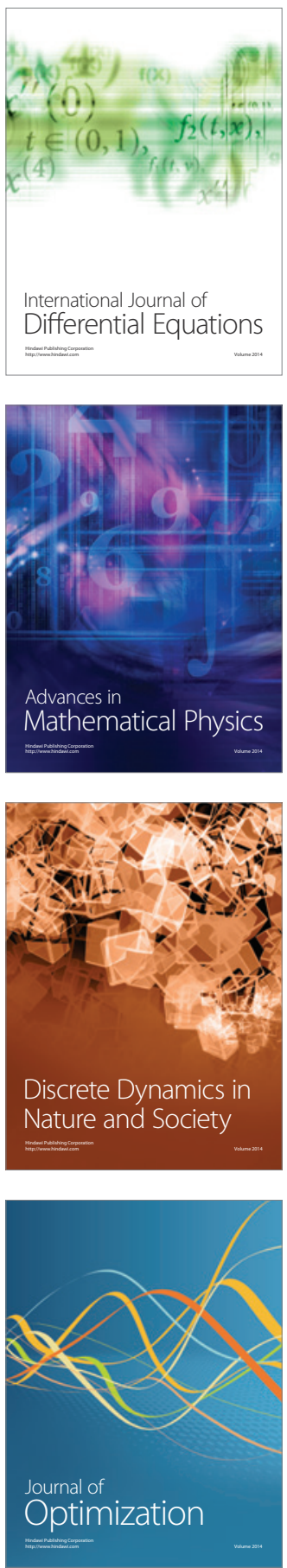